\title{
Transparent organic bistable memory device with pure organic active material and Al/indium tin oxide electrode
}

\author{
Kyoung Soo Yook, ${ }^{1}$ Jun Yeob Lee, ${ }^{1, a)}$ Sung Hyun Kim, ${ }^{2}$ and Jyongsik Jang ${ }^{2}$ \\ ${ }^{1}$ Department of Polymer Science and Engineering, Dankook University, Jukjeon-dong, Suji-gu, Yongin-si, \\ Kyeonggi-do 448-701, Republic of Korea \\ ${ }^{2}$ School of Chemical and Biological Engineering, Seoul National University, Shinlim-dong, Kwanak-gu, \\ Seoul 151-742, Republic of Korea
}

(Received 8 April 2008; accepted 10 May 2008; published online 3 June 2008)

\begin{abstract}
Transparent organic bistable memory devices (OBDs) were developed by employing indium tin oxide (ITO) as an anode and a cathode for OBD. A cathode structure of aluminum (Al)/ITO was used and bistability could be realized with pure polyphenylenevilylene based polymer active material without any metal nanoparticle. Transmittance of over 50\% could be obtained in Al/ITO based OBD at an Al thickness of $10 \mathrm{~nm}$, and an average on/off ratio around 100 was observed. (C) 2008 American Institute of Physics. [DOI: 10.1063/1.2938878]
\end{abstract}

Organic bistable memory devices (OBDs) have many advantages over current Si based memory devices such as simple fabrication process, high on/off ratio, design versatility, fast switching speed, and so on. In addition, OBDs can be used in flexible electronic system due to their flexibility in OBDs. ${ }^{1}$

There have been many studies about OBDs for the past five years and the most effective method to realize OBDs was to adopt a composite of organic and metal nanoparticle (NP). ${ }^{2-9}$ Metal NPs in organic layer were found to be essential to get high on/off ratio and long-term stability. Quyang et al., Bozano et al., and Yang et al. fabricated OBDs with organic-metal NP composite by codeposition of organic materials and $\mathrm{Al}^{2-4}$ Solution type nanocomposite of polymergold $(\mathrm{Au}) \mathrm{NP}$ was also used as an active material for OBD and thiol capped Au NP could give good memory characteristics. ${ }^{5-9}$ However, reproducibility of the memory performances of metal NP incorporated devices could not be guaranteed due to nonuniform dispersion of NP in organic matrix. An alternative for metal NP incorporated OBDs is to adopt pure organic materials. ${ }^{10-18}$ Although memory performances were not as good as those of metal NP embedded OBDs, bistability was observed. Even though bistability mechanism is not clear yet, oxide formation during aluminum (Al) evaporation was proposed as the main mechanism for bistability and filament conduction mechanism was suggested. ${ }^{19,20}$

OBDs are promising as a memory device and they can be used in combination with organic light-emitting diodes. Transparent electrodes are required for display applications and transparent OBDs should be developed for their use in organic display. Transparent OBDs may be realized by using indium tin oxide (ITO) as an anode and a cathode, but memory performances cannot be good enough because there is no aluminum oxide $\left(\mathrm{Al}_{2} \mathrm{O}_{3}\right)$ formation induced by cathode deposition. Therefore, metals have to be incorporated in electrode structure and we developed OBDs with a transparent electrode structure of Al/ITO. The relationship between the

\footnotetext{
a) Author to whom correspondence should be addressed. Electronic mail:
} leej17@dankook.ac.kr. Tel.: 82-31-8005-3585. FAX: 82-31-8005-3585. electrode structure and the memory performances was studied in this work.

It is essential to use transparent electrodes to fabricate transparent OBDs and ITO can be used both as a bottom electrode and a top electrode in transparent OBDs. In this work, four different device structures with ITO bottom electrode and different top electrodes have been investigated. A glass substrate with $150 \mathrm{~nm}$ thick ITO was used as a substrate for OBDs, and a pixel area was $4 \mathrm{~mm}^{2}$ (2 mm $\times 2 \mathrm{~mm}$ ). Device I used a $100 \mathrm{~nm}$ thick Al top electrode layer, while device II had an ITO top electrode. Devices III and IV had Al/ITO as a top electrode, where ITO was sputtered on Al. The Al electrode can be used as a semitransparent electrode in OBDs, if its thickness is smaller than $10 \mathrm{~nm}$. Therefore, the thicknesses of the Al electrode in devices III and IV were controlled to be 5 and $10 \mathrm{~nm}$, respectively. The active material was polyphenylenevinylene (PPV) based Superyellow ${ }^{\circledR}$ supplied from Merck, and the thickness of the active layer was $100 \mathrm{~nm}$. Electrical measurements of OBDs were carried out using Keithley 2400 source measurement unit after encapsulation of OBDs.

Figure 1 shows the current density-voltage characteristics of devices I and II. Device I with the Al electrode shows a hysteresis during the forward and backward scans, while device II does not show any hysteresis during forward and backward scans. In previous work, OBDs with PPV active layer and thermally deposited Al electrode showed bistable memory characteristics. ${ }^{17}$ Similar results were obtained in our work and it might be due to $\mathrm{Al}_{2} \mathrm{O}_{3}$ formation during $\mathrm{Al}$ evaporation. ${ }^{19,20}$ Compared to OBDs with $\mathrm{Al}$ electrode, ITO deposited OBDs did not show any bistability. Considering that metal oxide formation at the interface between $\mathrm{Al}$ and organic active layer is responsible for memory behavior in $\mathrm{Al}$ deposited OBDs, ${ }^{19,20}$ ITO deposition on PPV polymer cannot induce any metal oxide formation during sputter deposition, resulting in no bistable behavior during forward and backward scans. Therefore, ITO cannot be used as a transparent electrode in OBDs and it should be combined with $\mathrm{Al}$ to make transparent OBDs. Hence, an Al/ITO layered structure was deposited in order to fabricate transparent OBDs with stable memory performances. The $\mathrm{Al}$ deposition is required for metal oxide formation on top of the PPV layer and it should be used as a thin layer to get high transmittance. 

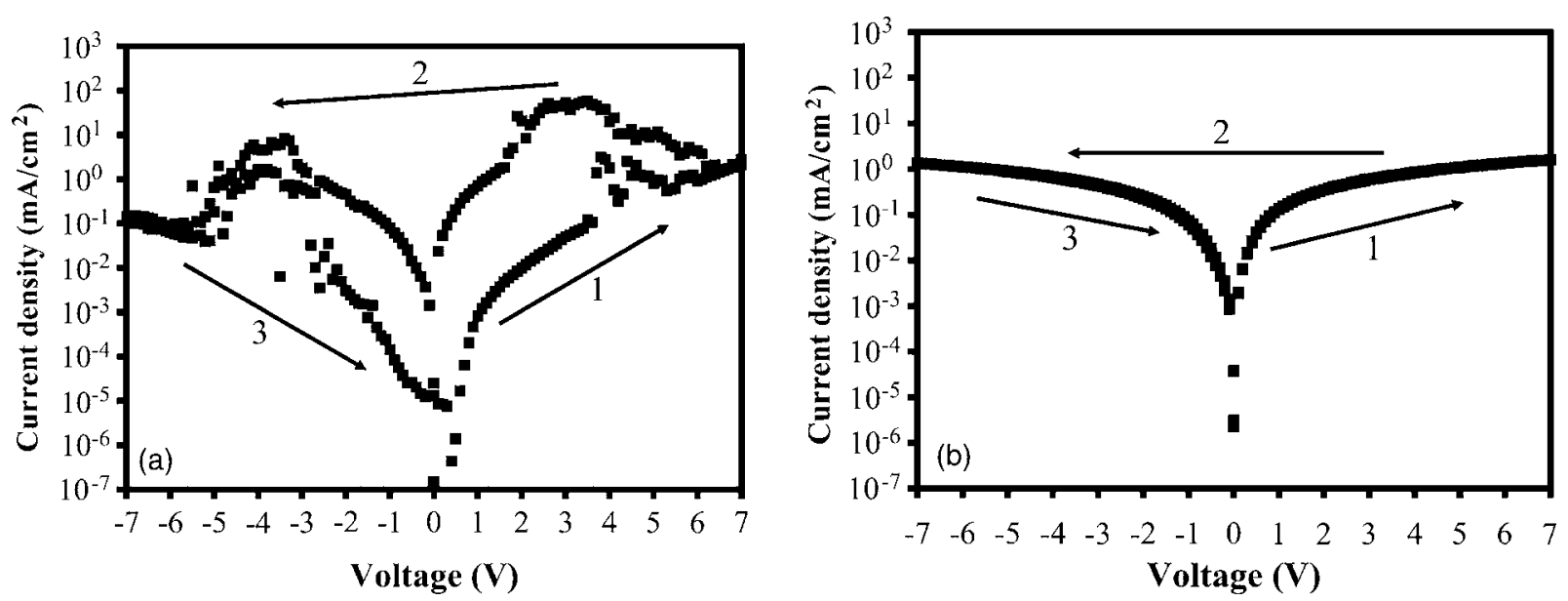

FIG. 1. Current density-voltage characteristics of a thermally deposited Al device [(a), device type I] and of a ITO sputtered device [(b), device type II].

Figure 2 represents current density-voltage curves of $\mathrm{Al} /$ ITO devices according to the thickness of the Al layer. $\mathrm{Al}(5 \mathrm{~nm}) / \mathrm{ITO}$ device did not show any bistability in the current density-voltage curves, while the $\mathrm{Al}(10 \mathrm{~nm}) / \mathrm{ITO}$ device exhibited bistable behavior between 0 and $5 \mathrm{~V}$. A negative differential resistance behavior and a hysteresis between forward and backward scans were observed. The high current state corresponds to high conductance state (on state) in the memory device, while the low current state characterizes low conductance state (off state). The memory device can be operated between 1 and $4 \mathrm{~V}$ and the on/off ratio of memory device was around 100 at a reading voltage of $2 \mathrm{~V}$. The write voltage of $\mathrm{Al}(10 \mathrm{~nm}) / \mathrm{ITO}$ device could be set as $5 \mathrm{~V}$, while a voltage of $-5 \mathrm{~V}$ can be used as an erase signal. Bistable memory behavior could be realized by using a pure organic material without any metal NPs. The development of the high conductance state in the $\mathrm{Al}(10 \mathrm{~nm}) / \mathrm{ITO}$ device may be explained by the formation of $\mathrm{Al}_{2} \mathrm{O}_{3}$ during the Al/ITO electrode deposition process. ${ }^{19,20}$ Partial oxidation of $\mathrm{Al}$ during deposition is known to be induced by residual oxygen inside the deposition chamber and the $\mathrm{Al}_{2} \mathrm{O}_{3}$ may be responsible for the bistability as reported by Verbakel et al. ${ }^{19}$ The disappearance of memory behavior in the OBD with $\mathrm{Al}(5 \mathrm{~nm}) / \mathrm{ITO}$ electrode indicates that a $5 \mathrm{~nm}$ thick $\mathrm{Al}$ layer is not sufficient for bistability causing metal oxide formation at the interface. In previous work, memory behavior was not clear in the device with thin $\mathrm{Al}_{2} \mathrm{O}_{3}$ layer. ${ }^{19}$

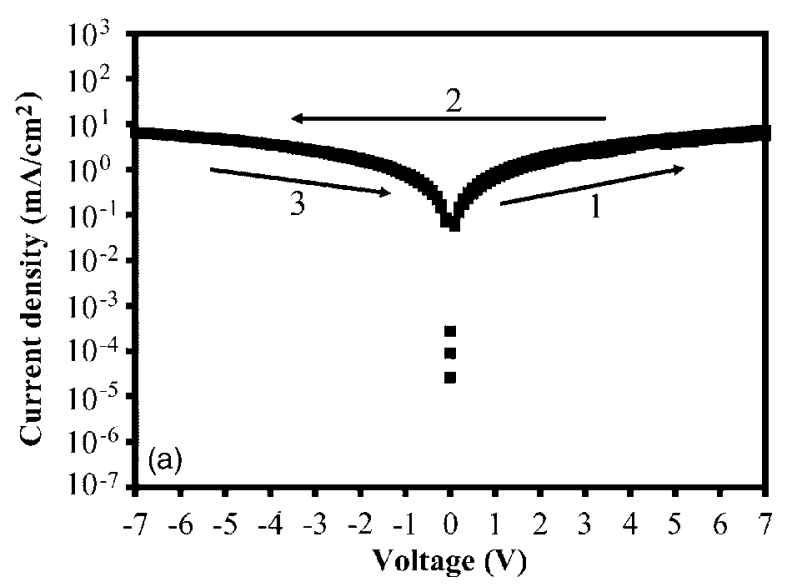

To investigate charge transport mechanism of PPV based OBDs, current density and voltage were plotted in log scale (Fig. 3). There, a linear relationship between current density and voltage is observed, which indicates that space charge limited conduction is dominant for charge transport at both conductance states. ${ }^{21}$ Space charge formation at the interface between PPV and $\mathrm{Al}$ may be induced by $\mathrm{Al}_{2} \mathrm{O}_{3}$, resulting in space charge limited current behavior in PPV OBDs with Al/ITO electrode. A similar result was already reported by Chen et al. in OBDs with Ag electrode and an anthracene derivative as the active material. ${ }^{16}$ We observe the space charge limited current behavior in the PPV OBD with $100 \mathrm{~nm}$ thick Al electrode (device I) too. Therefore, we conclude that ITO deposition on Al did not change the charge transport mechanism of PPV based OBDs. Compared with devices I and IV, a linear relationship between the current density and voltage was not observed even though data are not shown here.

The bistable performances of the transparent OBD with an Al/ITO electrode (device IV) were tested under cyclic voltage sweeps, using the same writing, erasing, and reading voltages as described above. The cycled on/off current data of the transparent OBD are shown in Fig. 4. Even though there was some scattering of the current level, an average on/off ratio of 100 was observed. Therefore, it can be concluded that metal NP free transparent OBD with Al/ITO deposited electrode can be used as a bistable memory device.

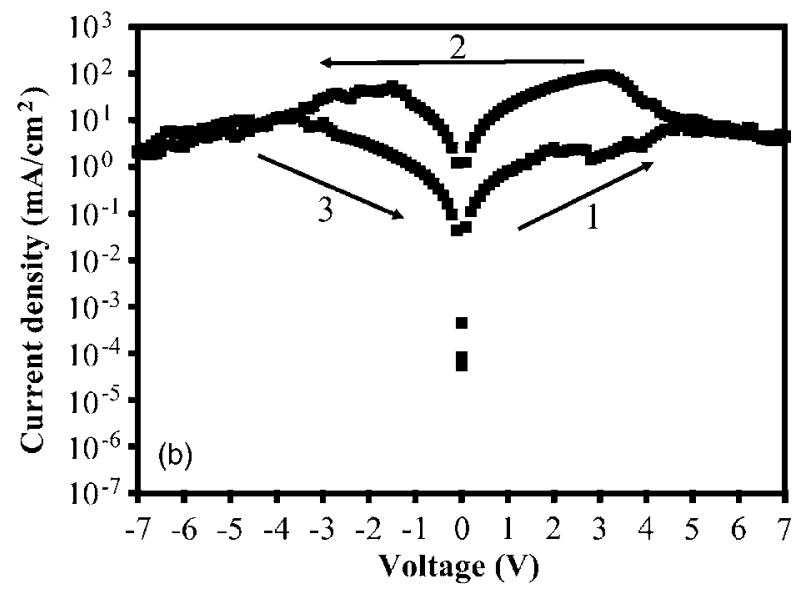




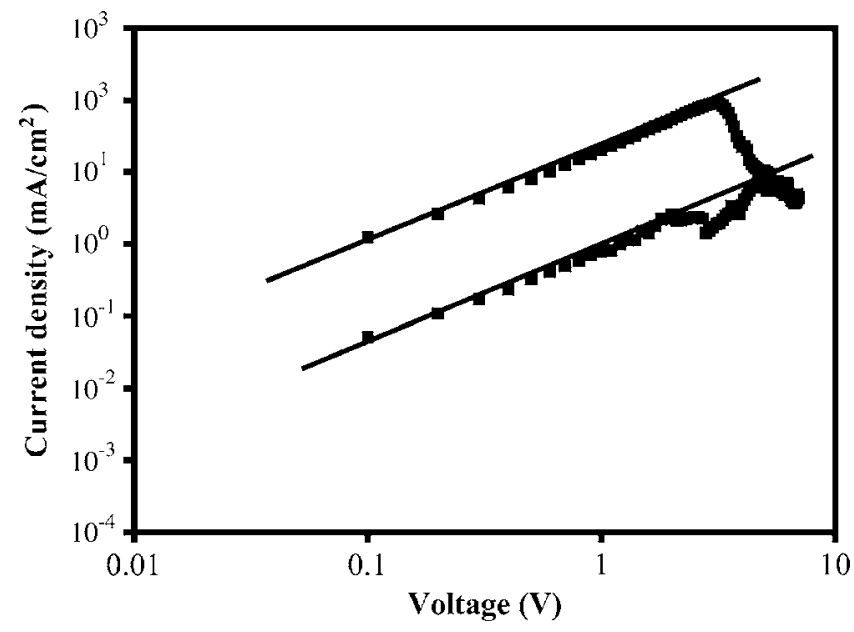

FIG. 3. $\log$ (current density)- $\log ($ voltage) plot recorded for a transparent organic memory device with $\mathrm{Al}(10 \mathrm{~nm}) /$ ITO electrode (device IV).

A picture of a transparent organic memory device with $\mathrm{Al}(10 \mathrm{~nm}) / \mathrm{ITO}$ electrode (device IV) is shown in Fig. 5. A pixel area was indicated as a square box with $2 \times 2 \mathrm{~mm}^{2}$ size. The pixel area of the device was transparent, and the symbol of Dankook University under the device is quite clear in this picture. The transmittance of the device was $51 \%$ at a wavelength of $550 \mathrm{~nm}$. Therefore, due to high transmittance, the transparent OBD developed in this work can be combined with organic light-emitting displays for future applications such as OBD driven light-emitting devices.

In summary, metal NP free pure organic based transparent OBDs with high transmittance could be effectively fabricated by using $\mathrm{Al}(10 \mathrm{~nm}) / \mathrm{ITO}$ as an electrode in OBDs. Hysteresis was observed during forward and backward scans, and a high on/off ratio of 100 was obtained. In addition, the space charge limited conduction mechanism dominates the charge transport in transparent OBDs with Al/ITO double layer electrode.

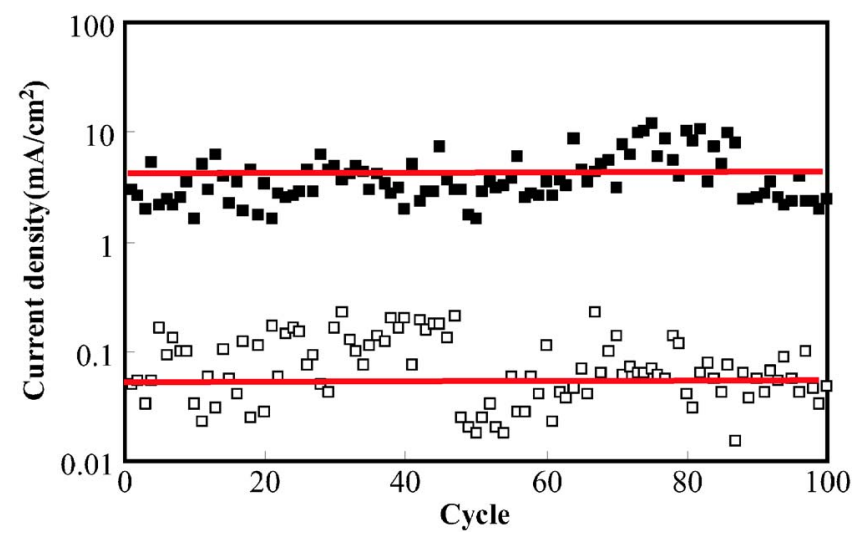

FIG. 4. (Color online) Cycled on-off test of transparent organic memory device with $\mathrm{Al}(10 \mathrm{~nm}) / \mathrm{ITO}$ electrode (device IV).

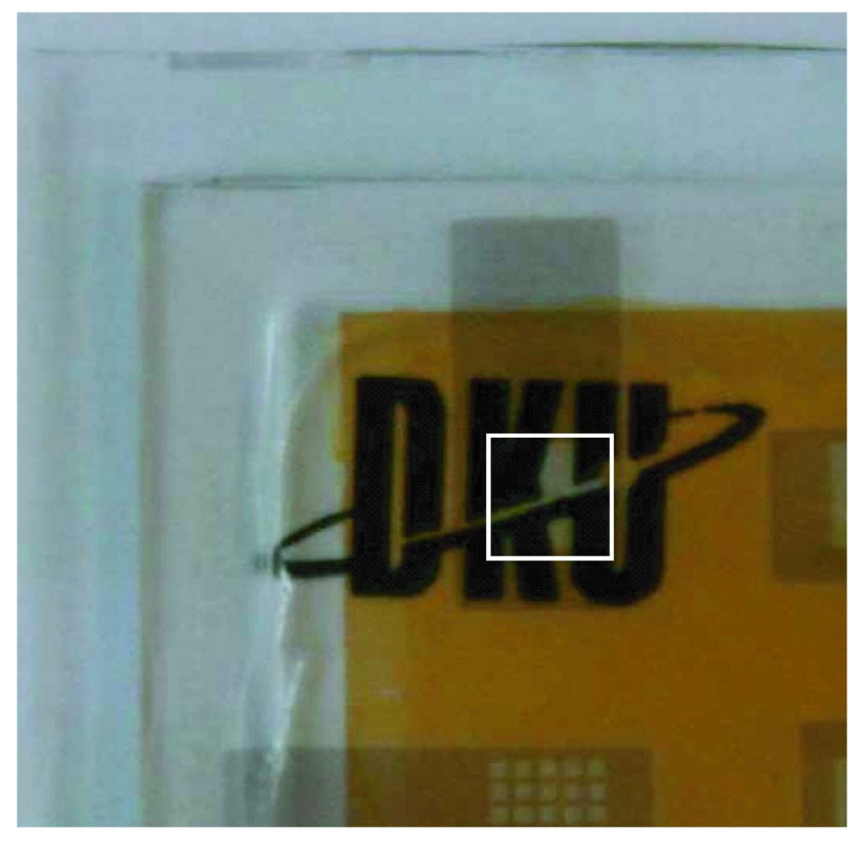

FIG. 5. (Color online) Picture of transparent organic memory device with $\mathrm{Al}(10 \mathrm{~nm}) / \mathrm{ITO}$ electrode (device IV). A pixel area was indicated as a square box in the main text.

${ }^{1}$ L. Li, Q. Ling, S. Lim, Y. Tan, C. Zhu, D. S. H. Chan, E. Kang, and K. Neoh, Org. Electron. 8, 401 (2007).

${ }^{2}$ J. Quyang, C. Chu, C. R. Szmanda, L. Ma, and Y. Yang, Nat. Mater. 3, 918 (2003).

${ }^{3}$ L. D. Bozano, B. W. Kean, M. Beinhoff, K. R. Carter, P. M. Rice, and J. C. Scott, Adv. Funct. Mater. 15, 1933 (2005).

${ }^{4}$ Y. Yang, J. Quyang, L. Ma, R. J. Tseng, and C. Chu, Adv. Funct. Mater. 16, 1001 (2006).

${ }^{5}$ R. J. Tseng, J. Huang, J. Quyang, R. B. Kaner, and Y. Yang, Nano Lett. 5, 1077 (2005).

${ }^{6}$ J. Quyang, C. Chu, D. Sieves, and Y. Yang, Appl. Phys. Lett. 86, 123507 (2005).

${ }^{7}$ A. Prakash, J. Quyang, J. Lin, and Y. Yang, J. Appl. Phys. 100, 054309 (2006).

${ }^{8}$ H. P. Wang, S. Pigeon, R. Izquierdo, and R. Martel, Appl. Phys. Lett. 89, 183502 (2006)

${ }^{9}$ C. Tu, D. Kwong, and Y. Lai, Appl. Phys. Lett. 89, 252107 (2006).

${ }^{10}$ A. Kanwal and M. Chhowalla, Appl. Phys. Lett. 89, 203103 (2006).

${ }^{11}$ V. Meunier, S. V. Kalinin, and B. G. Sumpter, Phys. Rev. Lett. 98, 056401 (2007).

${ }^{12}$ J. Chen and D. Ma, J. Appl. Phys. 100, 034512 (2006).

${ }^{13}$ J. Chen and D. Ma, Appl. Phys. Lett. 87, 023505 (2005).

${ }^{14}$ Y. Lai, C. Tu, D. Kwong, and J. S. Chen, Appl. Phys. Lett. 87, 122101 (2005).

${ }^{15}$ M. Lauters, B. McCarthy, D. Sarid, and G. E. Jabbour, Appl. Phys. Lett. 87, 231105 (2005).

${ }^{16}$ J. Chen, L. Xu, J. Lin, Y. Geng, L. Wang, and D. Ma, Appl. Phys. Lett. 89, 083514 (2006).

${ }^{17}$ M. Lauters, B. McCarthy, D. Sarid, and G. E. Jabbour, Appl. Phys. Lett. 89, 013507 (2006).

${ }^{18}$ T. Ouisse and O. Stephan, Org. Electron. 5, 251 (2004).

${ }^{19}$ F. Verbakel, S. C. J. Meskers, and R. A. J. Janssen, H. L. Gomes, M. Cölle, M. Büchel, and D. M. de Leeuw, Appl. Phys. Lett. 91, 192103 (2007).

${ }^{20}$ M. Cölle, M. Büchel, and D. M. de Leeuw, Org. Electron. 7, 305 (2006).

${ }^{21}$ M. A. Lampert and P. Mark, Current Injection in Solids (Academic, New York, 1970). 